\title{
A Rarely Seen Breast Tumor: Looking Over the Literature Related to Intracystic Papillary Carcinoma
}

\author{
Feryal KARACA, ${ }^{1}$ Çetin BÜLENT, ${ }^{2}$ Betül KÜÇÜKZEYBEK BOLAT ${ }^{3}$ \\ 'Department of Radiation Oncology, Adana Numune Training and Research Hospital, Adana-Turkey \\ ${ }^{2}$ Department of Medical Oncology, Recep Tayyip Erdoğan University Faculty of Medicine, Rize-Turkey \\ ${ }^{3}$ Department of Medical Pathology, İzmir Katip Çelebi University Faculty of Medicine, İzmir-Turkey
}

\section{Introduction}

Tumors of intramedullary papillary tumors are very rare tumors. They are $3 \%$ of all breast cancers.[1] $\mathrm{Pa}$ tients usually come from the mammary mass, from the nipple discharge or radiological abnormality.

Ductal carcinoma is treated as a variation of insitunun. However, intracystic papillary carcinomas differ clinically and radiologically from ductal carcinoma insitu.

Intraclavicular papillary carcinoma is usually surrounded by a fibrous capsule and is limited within the capsule. Histologically, dilate ductus arises and grows there. Malignant papillary lesions are associated with the largest change in breast pathology.

The first intracavitary papillary carcinoma case was shown in 1962 by Kraus and Neumbecker.[2] Subsequently, Carter and his friends found that the papillary variant's intracystic papular carcinoma and ductal carcinoma inset were classified as intraductal malignant lesions.[3]

Intracystic papillary carcinoma is diagnosed immunohistochemically in the papillary cell layer by the absence of myoepithelial cell layer. Characteristically, it is diagnosed with anti-p63, smooth muscle actin and CD10 antibody.[4,5] Intracystic papillary carcinoma of the chest is a very rare tumor with very few revivities and few cases are encountered. Our aim in this study is to differentiate between our approach in terms of illness treatment.

\section{Case Report}

Postmenopausal 51 year-old female patient had a massive cystic lesion on the right side of the left mammal at 3 oclock on the left mammary gland with a dense con- 

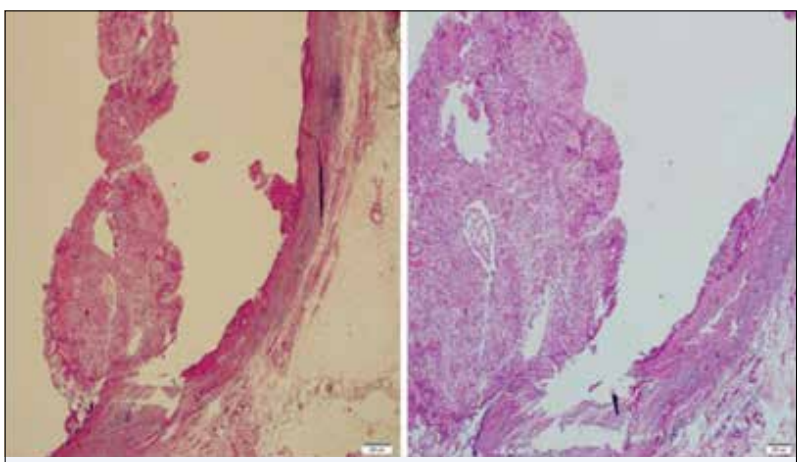

Fig. 1. Neoplastic cells (H \& Ex 20, H \& amp; Ex40) showing papillary proliferation within the fibrous capsule.

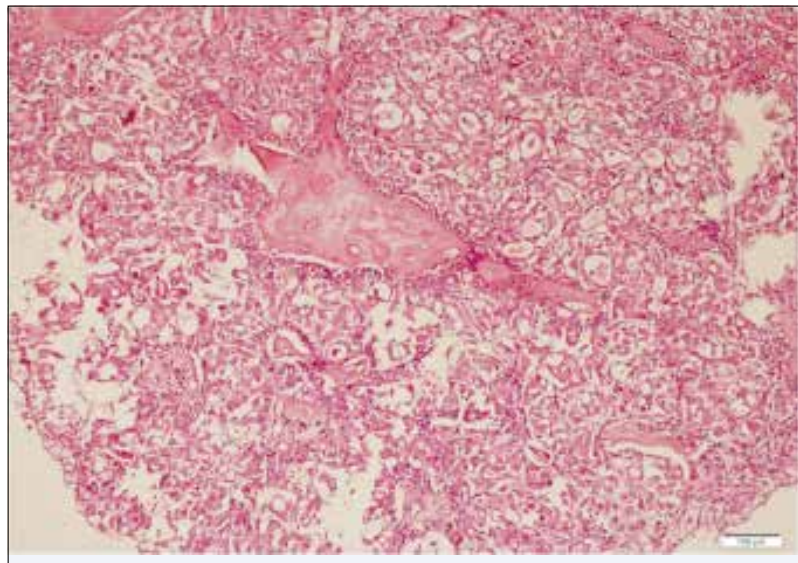

Fig. 2. Papillaries surrounded by a single uniform cell population without myoepithelial cells (H\&Ex 100).

tent thick wall structure containing solid components $15 \times 12 \mathrm{~mm}$ in size at $1 \mathrm{~cm}$ distance.

Histopathological diagnosis is recommended. On mammogram, a mass image with a contour of $15 \mathrm{~mm}$ in diameter with a contour was observed in the internal mammogram. On the upper external mammogram of the right breast, a uniformly limited image of the dens with a diameter of $5 \mathrm{~mm}$ was observed (intramammarial LAP?). Bx was taken at the hospital where the patient referred, but no pathological diagnosis was made. Then the breast of the patient was excised in the outer center.

Afterwards, exterior center pathology of exclusion a tumor with papillomatous areas on the inner surface of the esophageal pathology (İzmir Katip Çelebi University, Atatürk Training and Research Hospital Pathology No: 2014 / P-27016) with dimensions of $2.7 \times 2.5 \times 2.1 \mathrm{~cm}$ was serous hemorrhagic fluid within the cystic sections (Figure 1).

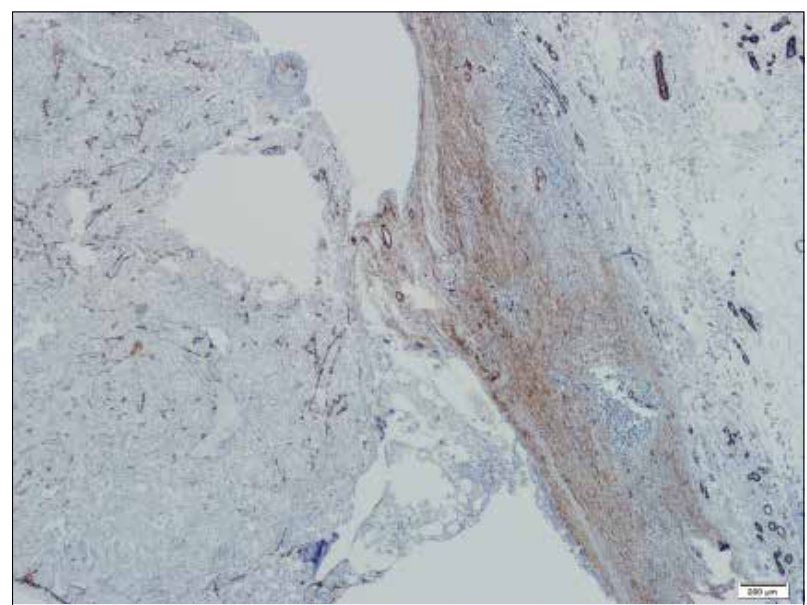

Fig. 3. Smooth muscle actinx40.

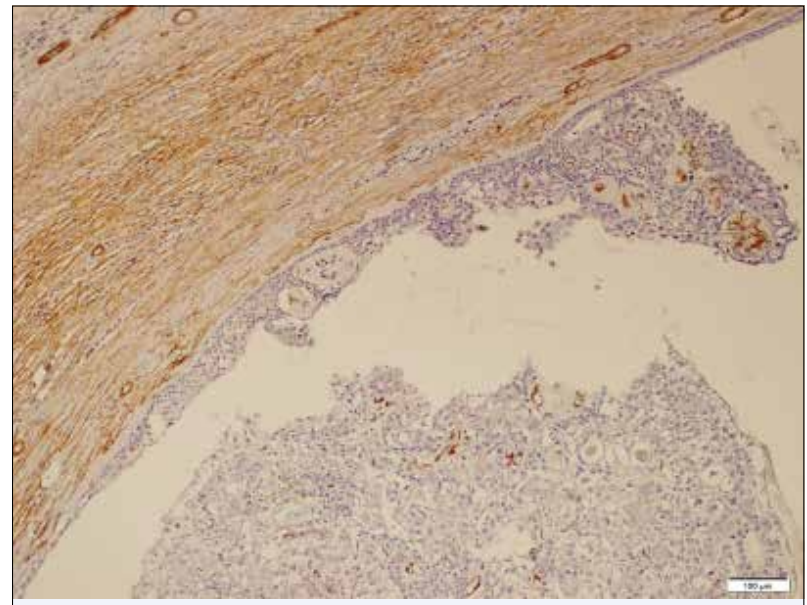

Fig. 4. Smooth muscle actinx100). Immunohistochemical examination Absence of myoepithelial cells in papillary and periphery of smooth muscle actin stain. Neighboring normal ducts and lobules are surrounded by smoothmuscle actin-positive myoepithelial cells.

Tumor diameter $2.7 \mathrm{~cm}$ tumor is nuclear grade 2 . Surgical limits are released. Lateral surgery revealed low nuclear grade ductal carcinoma insitu focus at 2 $\mathrm{mm}$ distance. In the area of intracystic papillary carcinoma, ER (-), PR\% (-), CERB-2 (-), cytokeratin14 (+), cytokeratin AE1 / AE3 (+), P53 5\% and KI-67 proliferation index were reported as $3 \%$.

In addition, smooth muscle actin and calponin applied in this focus were found in the tumor and in the periphery (-) (Figures 2-4). The papillary ductal carcinoma in situ was ER 20\% (3+), PR 15\% (2+) and smooth muscle actin and calponin were detected in peripheral myoepithelial cells (Figure 5). 


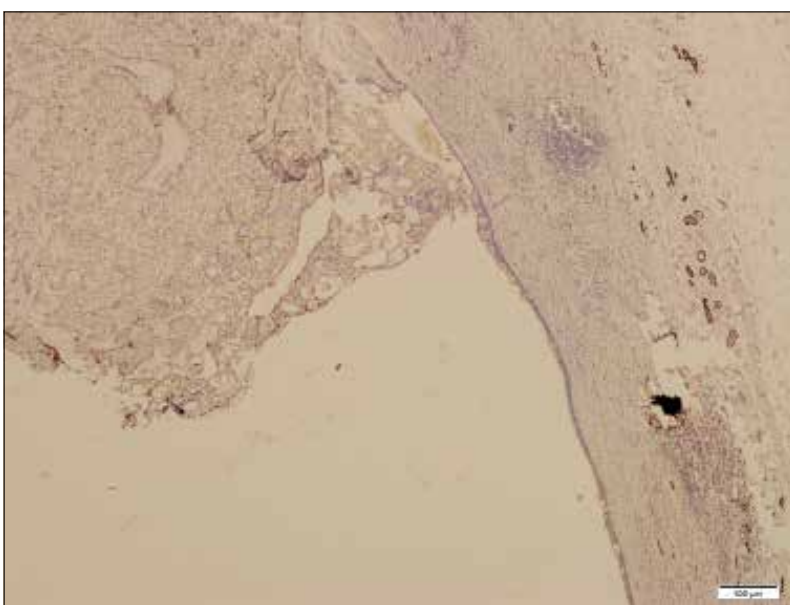

Fig. 5. Absence of myoepithelial cells in the papillae and periphery of the lesion with calponin dye in the immunohistochemical examination. Neighboring normal ducts and lobules are surrounded by positive myoepithelial cells (Calponinx40).

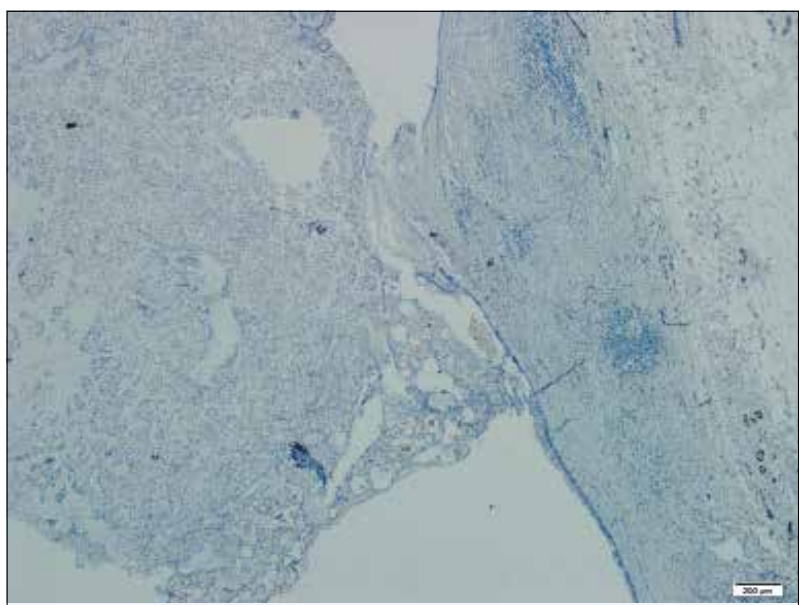

Fig. 6. Immunohistochemical examination revealed negative in ER and tumor cells (ERX40).

In addition, a block of smooth muscle actin, calponin, P63, cytokeratin 5/6 was administered; No diagnostic staining pattern was observed with cytokeratin $5 / 6$ and p63 (Figures 6,7 ). The patient was diagnosed as intracystic papillary carcinoma (encapsulated papillary carcinoma).

Then, reactive lymph node hyperplasia was detected in 21 lymph nodes after the axillary dissection of the patient. As a result of council, it was deemed appropriate to take $\mathrm{KT}+\mathrm{RT}+\mathrm{HT}$ in the next treatment of the patient. The patient was admitted to our clinic to receive 4 cycles of Adriamycin + Cyclophosphamide.



Fig. 7. Immunohistochemical examination in CerBB2 negative in tumoral cells (CerbB2x200).

The patient received curative doses of radiotherapy and hormonal therapy after chemotherapy. No local recurrence or metastasis were detected at the 40-month follow-up.

Immunohistochemical examination Absence of myoepithelial cells in papillary and periphery of smooth muscle actin stain. Neighboring normal ducts and lobules are surrounded by smooth muscle actinpositive myoepithelial cells (Figure 3: smooth muscle actin $x 40$, Figure 4: smooth muscle actinx100)

\section{Discussion}

Breast cystic formations are very common. Slowly growing cystic lesions seen in postmenopausal patients should be followed up. Carcinomas seen in the fibrous tissue surrounding the cyst are rare.

The majority of intracystic carcinomas are intracystic papillary carcinomas and the remaining pathologies are invasive ductal carcinoma with cystic degeneration, medullary carcinoma, mucinous carcinoma, adenoid cystic carcinoma and squamous carcinoma.[6] Intracystic carcinomas occur in $0.5 \%$ to $1 \%$ of female breast carcinomas.

They consist of a single cyst or a few cysts in the retroaerolar region of $70-90 \%$ and are placed in a single quadrant. 5-25\% may have nipple discharge and $18 \%$ of cases are asymptomatic. Intracystic papillary carcinoma is seen as a round or oval mass on mammogram. Indefinite limited and spicially contoured appearance is rare. Mammograms can be seen in areas of microcalcification as well as in the immune system. Fine needle aspiration biopsy or true-cut biopsy is the most difficult to identify. 
Solorzano and his colleagues therefore said that an excisional biopsy was needed. The advanced carcinoma focus on a cystic wall may not manifest itself with this fine needle biopsy or cyst asphyxiation. An excisional biopsy is required to assess the entirety of the material. [7,8] Because intracavitary papillary carcinomas are rare, the treatment consensus is not clear. Carter et al.[3] noted that in patients treated with local excision in 1983, and with 7 intracystic papillary carcinomas without recurrence for 7 years, mastectomy patients were treated with additional treatment. Nowadays, segmental mastectomy is done with negative surgical margin in the treatment of intracystic papillary carcinoma in past history.[1,7]

In the recent studies, it was observed that there was no difference between the patients who underwent the segmental mastectomy and those who underwent the mastectomy and the recurrence and cancer-related deaths. Very rarely, axillary microinvasion, lymph node metastases, is encountered.

Wasserberg and colleagues found a $7 \%$ incidence of ductal carcinoma insitu intrapapillary carcinoma in the patient, with distant metastases in $2 \%$ of these patients with lymph node involvement. [8] Axillary lymph node dissection should be discussed after the sentinel lymph node sampling.[9]

Some studies suggest adjuvant radiotherapy and endocrine therapy. Evaluation of case-based treatment options is beneficial.[10] In our follow-up, cystectomy was performed after the axillary dissection and adjuvant treatments were given. No local recurrence or distant metastases were found during the 40- month follow-up of the patient. The patient was evaluated as complete remission.

\section{Result}

Cystic lesions seen in the mammals are benign formations. Intracystic papillary carcinoma of the memine is a very rare tumor and it is better than intraductal carcinoma. It is important that the cystic lesions seen in postmenopausal women are followed. Particular attention should be paid to the solid construction in the cyst. Cells that dysplase during localization or multiple foci on a wall of the cyst are rare. There is a risk of these lesions turning into intracystic papillary carcinoma over time. These structures in the cyst can develop papillary non invasive carcinoma. However, intracystic papillary carcinomas are slowly growing well-limited masses. Follow-up and case-based treatment protocols should be followed.

\section{Disclosure Statement}

The authors declare no conflicts of interest.

\section{References}

1. Fayanju OM, Ritter J, Gillanders WE, Eberlein TJ, Dietz JR, Aft R, et al. Therapeutic management of intracystic papillary carcinoma of the breast: the roles of radiation and endocrine therapy. Am J Surg 2007;194(4):497-500. Crossret

2. Kraus FT, Neubecker RD. The differential diagnosis of papillary tumors of breast. Cancer 1962;15:444-55.

3. Carter D, Orr SL, Merino MJ. Intracystic papillary carcinoma of the breast. After mastectomy, radiotherapy or excisional biopsy alone. Cancer 1983;52(1):14-9.

4. Hill CB, Yeh IT. Myoepithelial cell staining patterns of papillary breast lesions: from intraductal papillomas to invasive papillary carcinomas. Am J Clin Pathol 2005;123(1):36-44. Crossret

5. Tse GM, Tan PH, Lui PC, Gilks CB, Poon CS, Ma TK, et al. The role of immunohistochemistry for smoothmuscle actin, p63, CD10 and cytokeratin 14 in the differential diagnosis of papillary lesions of the breast. J Clin Pathol 2007;60(3):315-20. Crossret

6. Levine PH, Waisman J, Yang GC. Aspiration cytology of cystic carcinoma of the breast. Diagn Cytopathol 2003;28(1):39-44. Crossree

7. Solorzano CC, Middleton LP, Hunt KK, Mirza N, Meric F, Kuerer HM, et al. Treatment and outcome of patients with intracystic papillary carcinoma of the breast. Am J Surg 2002;184(4):364-8. Crossree

8. Wasserberg N, Morgenstern S, Schachter J, Fenig E, Lelcuk S, Gutman H. Risk factors for lymph node metastases in breast ductal carcinoma in situ with minimal invasive component. Arch Surg 2002;137(11):124952. Crossree

9. Akagi T, Kinoshita T, Shien T, Hojo T, Akashi-Tanaka $S$, Murata Y. Clinical and pathological features of intracystic papillary carcinoma of the breast. Surg Today 2009;39(1):5-8. Crossree

10. Grabowski J, Salzstein SL, Sadler GR, Blair S. Intracystic papillary carcinoma: a review of 917 cases. Cancer 2008;113(5):916-20. Crossre 